\title{
Development of standard for quantitative molecular characterization of dissolved organic matter by FT-ICR MS
}

\author{
CHEN HE ${ }^{1}$, YAHE ZHANG ${ }^{1}$, YUNYUN LI ${ }^{1}$, XIAOCUN
}

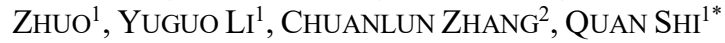

${ }^{1}$ China University of Petroleum, Beijing 102249, China

(hechen@cup.edu.cn, zhangyh@cup.edu.cn,

liyunyuncup@163.com, zhuoxiaocun@163.com, lyg18810060035@163.com,

*correspondence:sq@cup.edu.cn)

2 Southern University of Science and Technology, Shenzhen,

China (zhangcl@sustc.edu.cn)

Fourier transform ion cyclotron resonance mass spectrometry (FT-ICR MS) has been widely used for characterization DOM. However, FT-ICR MS generally has very poor repeatability and reproducibility because of its inherent structural characteristics, which severely limited its wide application on molecular composition analysis of complex mixtures. In this study, we developed a in-house standard for quantitative molecular characterization of DOM by FT-ICR MS. We abandoned the traditional method of fixing the operating parameters of the instrument and adopted the approach of object control to solve the problem of poor repeatability. The mass peak shape, resolution, and relative abundance distribution of a natural organic matter (NOM) standard were adjusted by optimizing the operating conditions to obatain a repeatable analysis result. A quality control sample was run 26 times by the different operators in a sixmonth long peirod to evaluate the reliability. Results showed that RSD\% of repeatability is 1.02 and 0.32 for average $\mathrm{H} / \mathrm{C}$ and double bond equivalent, respectively. The standard has been used for the characeterization of more than 4,000 samples and will be introduced to other laboratories. 